NOUVELle

\section{La rapamycine ouvre l'ère des thérapies ciblées dans les malformations veineuses}

Emmanuel Seront ${ }^{1}$, Nisha Limaye ${ }^{2}$, Laurence M. Boon ${ }^{2,3}$, Miikka Vikkula 2,3
${ }^{1}$ Institut Roi Albert II, département

d'oncologie médicale, cliniques universitaires Saint Luc, université catholique de Louvain, Bruxelles, Belgique ;

${ }^{2}$ Génétique Moléculaire Humaine, institut de Duve, université catholique de Louvain, Bruxelles, DDUV, Avenue Hippocrate 75, 1200 Woluwe-Saint-Lambert, Belgique; ${ }^{3}$ Centre des anomalies vasculaires, division de chirurgie plastique, cliniques universitaires Saint-Luc, université catholique de Louvain, Bruxelles, Belgique. miikka.vikkula@uclouvain.be

Implication du récepteur tyrosine kinase TIE2 et de la voie PI3K-

Akt-mTOR dans les malformations veineuses

Le récepteur tyrosine kinase TIE2 (tunica interna endothelial cell kinase 2), situé à la surface des cellules endothéliales, ainsi qu'un de ses ligands, l'angiopoiétine-1 (Angptl), sont des acteurs Bien que des formes familiales existent, les MV sont dans plus de $90 \%$ des cas sporadiques. Localisées ou diffuses, superficielles ou profondes, les MV peuvent survenir dans n'importe quelle partie du corps (Figure 1). Elles sont en général présentes à la naissance et grandissent progressivement durant la croissance de l'individu. Ces malformations veineuses, de par leur localisation et la compression des organes, provoquent des problèmes esthétiques, des douleurs chroniques, des oedèmes ou des saignements, résultant en une morbidité et une mortalité importante. Les MV prédisposent également à une stase veineuse ${ }^{4}$ entraînant un haut risque embolique. De plus, la moitié des MV étendues présente des défauts de coagulation (coagulopathie intravasculaire localisée) expliquée par des phénomènes thrombotiques entraînant une consommation des facteurs de coagulation. Actuellement, la sclérothérapie ${ }^{5}$, associée ou non à

\footnotetext{
${ }^{1}$ Déformées ou malformées.

${ }^{2}$ Dilatées.

${ }^{3}$ Présentant une anomalie de leur forme.

${ }^{4}$ Stagnation du sang au niveau d'une veine.

${ }^{5}$ La sclérothérapie est un traitement non invasif consistant en l'injection d'un produit qui va détruire la veine.
}

la chirurgie, constitue le traitement standard contre les MV. Malheureusement, ces traitements restent le plus souvent non curatifs et ils ne sont pas dénués de toxicité ; beaucoup de MV sont inaccessibles et/ou persistent après traitement local. II y a donc un besoin urgent de nouvelles stratégies thérapeutiques [1].
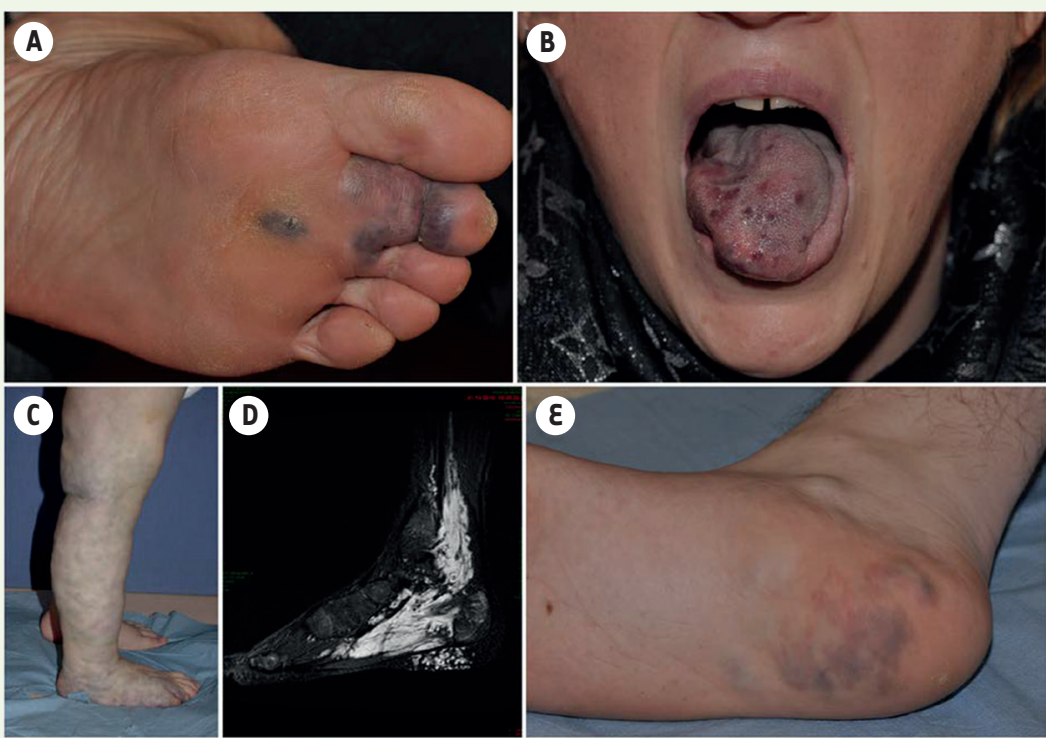

Figure 1. Aspects cliniques de malformations veineuses. A. MV localisée au niveau du pied. B. MV localisée dans la muqueuse de la langue, provoquant une apnée du sommeil. C. MV touchant les extrémités inférieures associées à une coagulopathie intravasculaire localisée sévère. $D-\varepsilon$. MV au niveau du pied et de la cheville avec atteinte musculaire, comme cela peut être observé par imagerie par résonance magnétique pondérée en T2 (visible en blanc). En résonance magnétique, la morphologie du signal émis par les protons dépend essentiellement du temps (appelé temps de relaxation) que ceux-ci mettent à revenir dans l'axe de l'aimant (temps $n^{\circ} 1$ ou $\mathrm{Tl}$ ) et du temps qu'ils mettent à se déphaser à nouveau (temps $n^{\circ} 2$ ou T2). Ces deux temps $\mathrm{Tl}$ et T2 sont propres à chaque type de tissu. En IRM T1, l'eau est noire et la graisse blanche. En IRM T2, l'eau est blanche et la graisse grise. MV : malformation veineuse. 


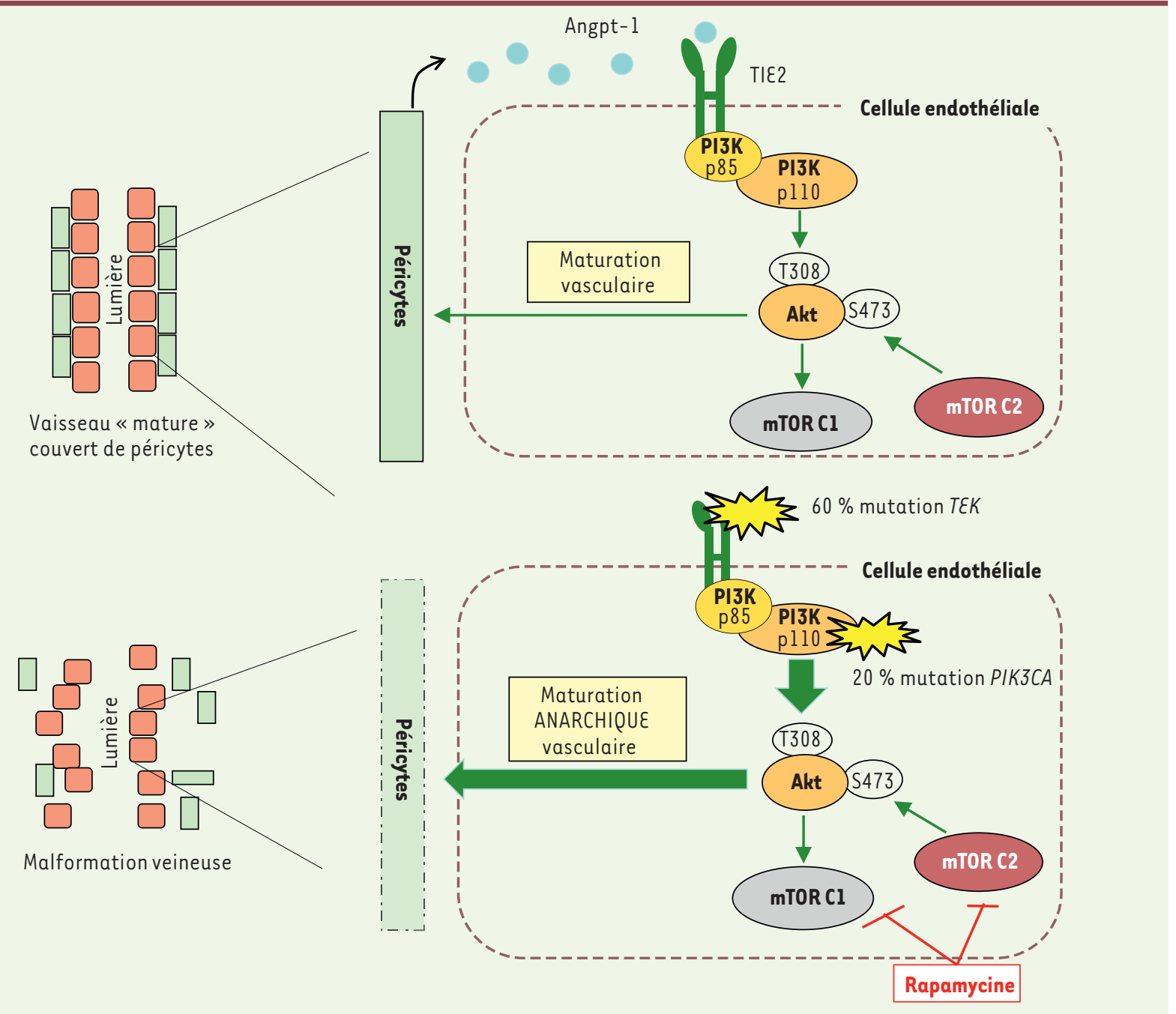

Figure 2. Voie de signalisation impliquée dans le processus de maturation vasculaire. $A$. Le récepteur tyrosine kinase TIE2 activé par un de ses ligands, l'angiopoiëtinel (ANGPT-1), stimule la voie PI3K-Akt. Akt, après une phosphorylation par PI3K et par mTORC2, régule positivement le processus de maturation vasculaire. B. Les mutations activatrices de TIE2 et de PIK3CA (mutations dans le gène TEK ou PIK3CA) induisent une stimulation excessive d'Akt résultant en la formation d'un réseau vasculaire dysfonctionnel. La rapamycine, en inhibant l'activité de mTORC2, empêche une activation excessive d'Akt et donc restaure la maturation vasculaire. PI3K : phosphoinositol 3 kinase; PIK3CA : domaine catalytique de PI3K ; mTORC2 : mammalian target of rapamycin complex 2.

indispensables de la formation, de la maturation et de la stabilité vasculaire (Figure 2A). Des souris déficientes en TIE2 ou Angptl développent en effet un réseau vasculaire dysfonctionnel et dépourvu de péricytes [2]. Ce rôle du récepteur TIE2 impliquerait la voie PI3KAkt-mTOR (phosphoinositide 3-kinase - protein kinase B - mammalian target of rapamycin), une cascade signalétique activée par différents récepteurs cellulaires, dont TIE2. Cette voie joue un rôle important dans la croissance et la prolifération des cellules. La protéine mTOR, au centre de cette cascade, existe sous la forme de deux complexes, le complexe 1 (mTORCl) et le complexe 2 (mTORC2). L'activation de TIE2 par son ligand entraîne, à la suite du recrutement de la sous-unité régulatrice p85 de la PI3K, l'activation de pl10, sa sous-unité catalytique. PI3K induit alors, par l'intermédiaire de différentes protéines, la phosphorylation d'Akt (sur la thréonine en position 308, Thr308). Une seconde phosphorylation (sur la sérine en position 473, Ser473) induite par mTORC2, est nécessaire pour qu'Akt active différents facteurs de transcription comme FOXOl (forkhead box protein 01) $[3,4]$.

Toute dérégulation du récepteur TIE2, ou de la voie PI3K-Akt, peut conduire au développement de malformations veineuses. Ainsi, les MV familiales, et plus de $60 \%$ des formes sporadiques, présentent des mutations du gène $T \varepsilon K$, codant le récepteur TIદ2. Ces mutations se traduisent par une activation 
soutenue de TIE2 indépendamment de sa liaison à ses ligands. La mutation de $T \varepsilon K$ la plus fréquemment retrouvée dans les formes familiales est la substitution $\mathrm{R} 849 \mathrm{~W}^{6}$, alors que dans les formes sporadiques, la mutation la plus fréquente (correspondant à $77 \%$ des cas) est $\mathrm{L} 14 \mathrm{~F}^{7}$ [5]. In vitro, les mutations de TIE2, exprimées dans des cellules endothéliales (HUVEC, human umbilical vein endothelial cells), entraînent une activation accrue d'Akt et de STAT- ${ }^{8}$ (signal transducer and activator of transcription 1) et une diminution de la production de PDGF-B (platelet-derived growth factor subunit B), un facteur de croissance stimulant la maturation endothéliale par attrait des péricytes autour des cellules endothéliales [6].

\section{Modèle animal de MV avec mutation de TIE2}

Pour la première fois, un modèle murin de MV similaires à celles rencontrées chez les patients, a pu être développé. Ce modèle a permis de confirmer le rôle des mutations du gène codant TIદ2 dans le développement des MV [7]. L'injection, dans des souris, de cellules HUVEC présentant un récepteur TIE2 muté (substitution L914F), en comparaison à des HUVEC portant un récepteur non muté (type sauvage, wild type $[\mathrm{wt}]$ ), induit la formation de lésions vasculaires à croissance rapide et composées de veines ectatiques (> $50 \mu \mathrm{m}$ de diamètre) et irrégulièrement couvertes de péricytes, à l'image des veines observées chez des patients atteints de MV. D'un point de vue fonctionnel, le débit sanguin dans ces lésions vasculaires reste très bas et non pulsatile comme pour les MV observées en clinique. Au niveau moléculaire, la mutation de TIદ2 est associée à une augmentation importante de l'activité d'Akt, confirmant le rôle du récepteur dans la stimulation de l'axe PI3K-Akt (Figure 2B).

\footnotetext{
${ }^{6}$ Substitution d'un tryptophane par une arginine en position 849 .

7 Substitution d'une phénylalanine par une leucine en position 914 .

${ }^{8}$ Facteur de transcription stimulé par l'interféron.
}

\section{MV et mutation de PI3K}

Un rôle des mutations de PI3K dans le développement des MV a également été mis en évidence chez l'homme [8]. En effet, une analyse moléculaire d'échantillons chirurgicaux provenant de 130 patients porteurs de MV localisées et uniques a permis de montrer qu'une mutation du gène PIK3CA, qui code le domaine catalytique de PI3K, est observée chez $20 \%$ de ces patients. Cette mutation est également retrouvée dans de nombreux cancers et est à l'origine d'une activation excessive d'Akt. Contrairement aux MV secondaires aux mutations du gène $T E K$, la présence d'une mutation PIK3CA induirait plutôt des MV ne touchant pas la peau. In vitro, la transfection du gène PIK3CA muté dans des cellules HUVEC entraîne, par rapport à des HUVEC exprimant le gène sauvage, une activation importante d'Akt et un dysmorphisme endothélial, comme observé dans les cellules HUVEC présentant le gène TIE2 muté. L'inhibition spécifique de PI3K par un inhibiteur pharmacologique permet de réduire l'activité d'Akt et de restaurer la morphologie cellulaire.

\section{La rapamycine comme traitement des} malformations veineuses

La rapamycine, appelée aussi sirolimus, est une molécule connue depuis plusieurs décennies; elle est extraite d'une algue récoltée sur l'île de Pâque (appelée aussi Rapa-Nui). Ses propriétés antimycotique, bactéricide, immunosuppressive et antiproliférative ont rapidement suscité l'intérêt des scientifiques. II aura cependant fallu près de 20 ans pour identifier la cible de la rapamycine, la protéine mTOR [9] $(\rightarrow)$, participant à la cascade signalétique PI3K-Akt-mTOR. La rapamycine agit en inhibant, de

\section{$(\rightarrow)$ Voir la Synthèse de L.A. Julien et P.P. Roux, $m / s$ $n^{\circ} 12$, décembre 2010 , page 1056} façon allostérique, la protéine mTOR. $\varepsilon$ n déstabilisant le complexe mTORC2, elle l'empêche ainsi de phosphoryler Akt et donc de l'activer [10] (Figure 2B). En thérapeutique, la rapamycine est utilisée couramment en pratique clinique comme immunosuppresseur pour la prévention des rejets de greffe et comme agent anticancéreux.

\section{Effet de la rapamycine sur un modèle pré-clinique}

Nous avons utilisé le modèle murin d'injection de cellules HUVEC porteuses d'un récepteur TIદ2 muté afin d'évaluer l'efficacité de la rapamycine dans la prévention et le traitement des MV [7]. Après injection de ces cellules, les souris ont été traitées soit par la rapamycine, soit par un inhibiteur de l'activité tyrosine kinase de TIE2, soit par une solution contrôle. Seul le traitement par rapamycine a empêché de façon significative la croissance et la vascularisation des cellules implantées et ce, par un effet spécifique sur les cellules endothéliales exprimant un récepteur TIE2 muté, indépendemment d'un éventuel effet sur les cellules murines. Morphologiquement, la rapamycine entraîne une réduction significative du diamètre des vaisseaux et une augmentation du nombre de péricytes. En plus de son effet sur la prévention du développement des MV, la rapamycine s'est également montrée efficace dans le traitement de MV bien établies chez la souris, en stoppant la progression de ces lésions [7].

\section{Effet de la rapamycine chez des patients humains}

Les résultats pré-cliniques obtenus chez la souris avec la rapamycine ont encouragé l'évaluation de cette molécule chez quelques patients porteurs de MV. Six patients atteints de $M V$ réfractaires aux traitements standards et très symptomatiques (présentant des douleurs, un déficit fonctionnel, des saignements, des infections récurrentes, un œdème, etc.) ont été traités par une prise orale quotidienne de rapamycine (2 mg par jour) en continu. Trois de ces patients étaient porteurs d'une mutation touchant le gène TEK, les trois autres présentaient une mutation du gène PIK3CA. 


\begin{tabular}{|c|c|c|c|}
\hline \multicolumn{4}{|c|}{ Efficacité clinique : Douleur $(0-10)$} \\
\hline $\begin{array}{l}\text { Ligne de base } \\
\text { Médiane (déviation standard) }\end{array}$ & 3 mois & 6 mois & 1 an \\
\hline $8,00(6,75-9,00)$ & $2,00(1,00-5,50)$ & $2,00(1,50-7,00)$ & $3,00(0,50-6,00)$ \\
\hline \multicolumn{4}{|c|}{ Efficacité biologique : D-dimères ${ }^{9}$ (négatif $<500 \mathrm{ng} / \mathrm{mL}$ ) } \\
\hline $\begin{array}{l}\text { Ligne de base } \\
\text { Médiane (déviation standard) }\end{array}$ & 3 mois & 6 mois & 1 an \\
\hline $6723(1685-12110)$ & $1934(920-2766)$ & $2562(677-4368)$ & $1085(884-1441)$ \\
\hline \multicolumn{4}{|c|}{ Effets secondaires } \\
\hline Effets secondaires : (n [\%]) & Total & Grade 1 - 2 & Grade 3 - 4 \\
\hline Fatigue & $2(33)$ & $2(33)$ & $0(0)$ \\
\hline Céphalée & $2(33)$ & $2(17)$ & $0(0)$ \\
\hline Stomatite & $3(50)$ & $2(33)$ & $1(17)$ \\
\hline Vomissement & $1(17)$ & $1(17)$ & $0(0)$ \\
\hline Diarrhée & $1 \quad(17)$ & $1 \quad(17)$ & $0(0)$ \\
\hline Syndrome grippal & $1 \quad(17)$ & $1 \quad(17)$ & $0(0)$ \\
\hline Neuropathie & $1 \quad(17)$ & $1 \quad(17)$ & $0(0)$ \\
\hline Édème & $1(17)$ & 1 (17) & $0(0)$ \\
\hline Rhinorrhée & $1 \quad(17)$ & $1(17)$ & $0(0)$ \\
\hline Arthralgie & $1 \quad(17)$ & $1(17)$ & $0(0)$ \\
\hline Vertige & $1(17)$ & $1(17)$ & $0(0)$ \\
\hline Rash cutané & $1 \quad(17)$ & $1(17)$ & $0(0)$ \\
\hline Sécheresse cutanée & $1 \quad(17)$ & $1 \quad(17)$ & $0(0)$ \\
\hline $\begin{array}{l}\text { Tumeur cutanée basocellulaire } \\
\text { (après l an) }\end{array}$ & $1(17)$ & $0 \quad(0)$ & $1(17)$ \\
\hline
\end{tabular}

Tableau I. Efficacité et effets secondaires de la rapamycine observés dans une étude pilote. La douleur est mesurée par une échelle (visual analogic scale), les patients devant évaluer l'intensité de leur douleur entre 0 (pas de douleur) et 10 (douleur la plus extrême possible). La mesure biologique correspond à un dosage sanguin des $D$-dimères. Les effets secondaires sont définis de façon standardisée telle que proposée par le NIH (National institute of health) dans le CTCAE v3 (common terminology criteria for adverse events v3) et leur intensité est évaluée par grades (grade 1 : léger; grade 2 : modéré ; grade 3 : sévère ; grade 4 : mettant la vie en danger) (d'après [7]).

Tous ces patients ont ressenti, dans le mois suivant le début du traitement, une diminution significative de la douleur et une amélioration de la qualité de
D-dimères ${ }^{9}$ ) se sont rapidement améliorés et les images radiologiques ont montré une diminution du volume des MV à $l$ an. Le traitement s'est montré de manière générale très bien toléré ; les effets secondaires les plus fréquents ont été des céphalées, des éruptions cutanées (rash), des aphtes et une diarrhée, mais tous de sévérité légère à modérée et tous réversibles par traitements symptomatiques. Un patient a cependant présenté, après un an, un carcinome basocellulaire, possiblement favorisé par l'effet immunosuppresseur de la rapamycine. Un autre patient a présenté une mucite ${ }^{10}$ sévère ayant nécessité un arrêt du traitement [7]. La rapamycine pourrait donc aujourd'hui constituer le premier traitement pharmacologique des MV réfractaires aux traitements standards. Faisant suite aux résultats cliniques encourageants que nous avons obtenus avec notre petit nombre de patients, une étude clinique de phase 3 multicentrique (NCT02638389) est actuellement en cours afin de confirmer l'efficacité de la rapamycine, ainsi que de s'assurer de sa bonne tolérance et de son innocuité à long terme. Cette étude permettra également de mieux préciser la place de la rapamycine dans la stratégie thérapeutique et d'identifier d'éventuelles caractéristiques cliniques et moléculaires prédictives d'une réponse thérapeutique. La rapamycine ouvre donc la voie au développement de nouvelles thérapies ciblées dans le domaine des MV. D'autres molécules interférant avec la voie PI3K-Akt-mTOR, comme des inhibiteurs spécifiques de PI3K ou d'Akt, pourraient également être investiguées à court terme afin de mieux cibler l'anomalie responsable des MV et de limiter les effets secondaires. $\diamond$

Rapamycin opens the era of targeted molecular therapy of venous malformations

vie (diminution des saignements et des œdèmes et amélioration fonctionnelle) (Tableau 1). Les paramètres biologiques de coagulation (évalués par dosage des
${ }^{9}$ Produits de la dégradation de la fibrine, une protéine impliquée dans la coagulation. Leur présence dans le sang peut être le signe de la formation d'un caillot sanguin.

${ }^{10}$ Inflammation des muqueuses. 


\section{LIENS D'INTÉRÊT}

Les auteurs déclarent n'avoir aucun lien d'intérêt concernant les données publiées dans cet article.

\section{RÉFÉRENCES}

1. Dompmartin A, Vikkula M, Boon LM. Venous malformation: update on aetiopathogenesis, diagnosis and management. Phlebology 2010 ; 25 : 224-35.

2. Suri C, Jones PF, Patan S, et al. Requisite role of angiopoietin-1, a ligand for the TIE2 receptor, during embryonic angiogenesis. Cell 1996; 87 : 1171-80.

3. Laplante M, Sabatini DM. mTOR signaling in growth control and disease. Cell 2012 ; 149 : 274-93.
4. Korpelainen El, Karkkainen M, Gunji Y, et al. Endothelial receptor tyrosine kinases activate the STAT signaling pathway: mutant Tie-2 causing venous malformations signals a distinct STAT activation response. Oncogene 1999 ; $18: 1-8$

5. Limaye N, Wouters V, Uebelhoer M, et al. Somatic mutations in angiopoietin receptor gene TEK cause solitary and multiple sporadic venous malformations. Nat Genet 2009 ; 41 : 118-24.

6. Uebelhoer M, Nätynki M, Kangas J, et al. Venous malformation-causative TIE2 mutations mediate an AKT-dependent decrease in PDGFB. Hum Mol Genet $2013 ; 22$ : 3438-48
7. Boscolo $\varepsilon$, Limaye $N$, Huang L, et al. Rapamycin improves TIE2-mutated venous malformation in murine model and human subjects. J Clin Invest 2015 $125: 3491-504$.

8. Limaye N, Kangas J, Mendola A, et al. Somatic activating PIK3CA mutations cause venous malformation. Am J Hum Genet 2015 ; 97 . 914-21.

9. Julien LA, Roux PP. mTOR, la cible fonctionnelle de la rapamycine. Med Sci (Paris) $2010 ; 26$ : 1056-60.

10. Sarbassov D, Ali SM, Sengupta S, et al. Prolonged rapamycin treatment inhibits $\mathrm{mTORC2}$ assembly and Akt/PKB. Mol Cell $2006 ; 22$ : 159-68.

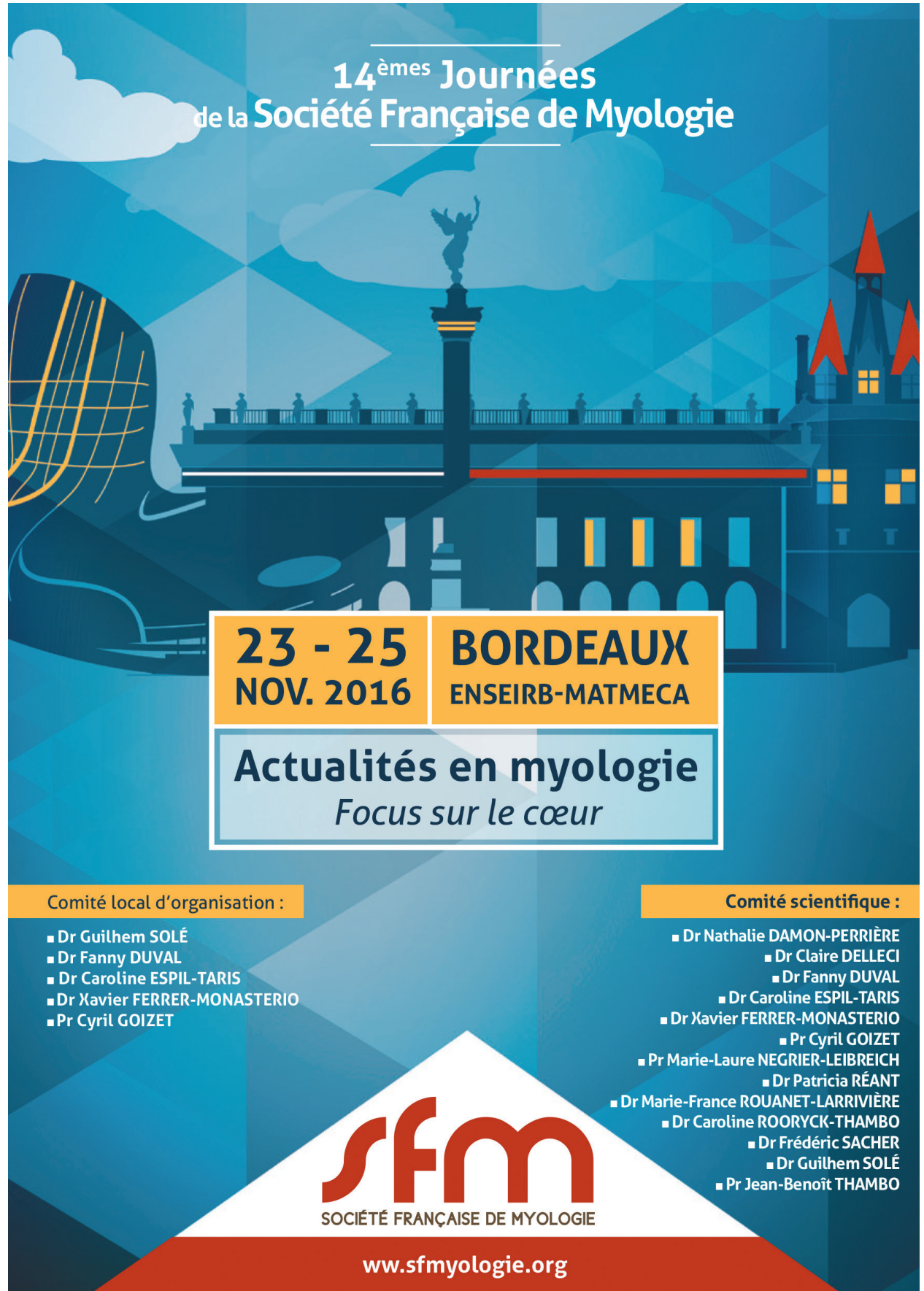

